\title{
Suplementação enteral e parenteral com glutamina em neonatos pré-termo e com baixo peso ao nascer
}

\author{
Maria Carolina Borges, Marcelo Macedo Rogero, Julio Tirapegui* \\ Departamento de Alimentos e Nutrição Experimental, Faculdade de Ciências Farmacêuticas, \\ Universidade de São Paulo
}

*Correspondência:

J. Tirapegui

Departamento de Alimentos e

Nutrição Experimental

Faculdade de Ciências Farmacêuticas

Universidade de São Paulo

Avenida Prof. Lineu Prestes, 580,

Bloco 14

05508-900 - São Paulo - SP, Brasil

E-mail: tirapegu@usp.br

\begin{abstract}
A glutamina é o aminoácido livre mais abundante no sangue e no músculo esquelético, bem como é o principal substrato energético para células de elevado turnover, como enterócitos e leucócitos. Adicionalmente, a glutamina representa o principal aminoácido transferido para o feto pela placenta e, juntamente com o glutamato, constituem os aminoácidos mais abundantes no leite materno. Todavia, bebês nascidos prematuramente sofrem interrupção abrupta do fornecimento placentário de glutamina, o que acarreta em dependência exclusiva da sintese endógena ou do fornecimento exógeno deste aminoácido. Aliado a isso, neonatos pré-termo (PT) e com baixo peso ao nascer (BPN), freqüentemente, recebem apenas nutrição parenteral total nas primeiras semanas de vida, a qual não contém glutamina. Cabe ainda destacar que esses bebês possuem pouca massa muscular e, portanto, seus estoques de glutamina são limitados. Uma vez que neonatos PT e com BPN estão sujeitos a intenso crescimento e a inúmeros estresses fisiológicos, é possível que a glutamina seja um nutriente condicionalmente essencial nessa fase da vida, fato que estimulou a realização de estudos com a finalidade de avaliar os possíveis benefícios clínicos da suplementação enteral e parenteral com glutamina em neonatos PT e com BPN.
\end{abstract}

\section{INTRODUÇÃO}

A glutamina é o aminoácido livre mais abundante no plasma e no tecido muscular, ao mesmo tempo em que apresenta diversas funções únicas no organismo, o que reforça seu papel relevante tanto em estados normais como fisiopatológicos (Figura 1) (Rogero et al., 2004). Este aminoácido é utilizado em altas taxas por células com eleva- do turnover, como enterócitos e leucócitos, para fornecer energia e favorecer a biossíntese de nucleotídeos, além de atuar tanto no anabolismo protéico, quanto na promoção do processo de adaptação e crescimento de tecidos altamente especializados (Neu et al., 1997; Ball, Hardy, 2002).

Dentre os órgãos envolvidos na síntese de glutamina, incluem-se o músculo esquelético, pulmões, fígado, cérebro e, possivelmente, o tecido adiposo, os quais contêm atividade 


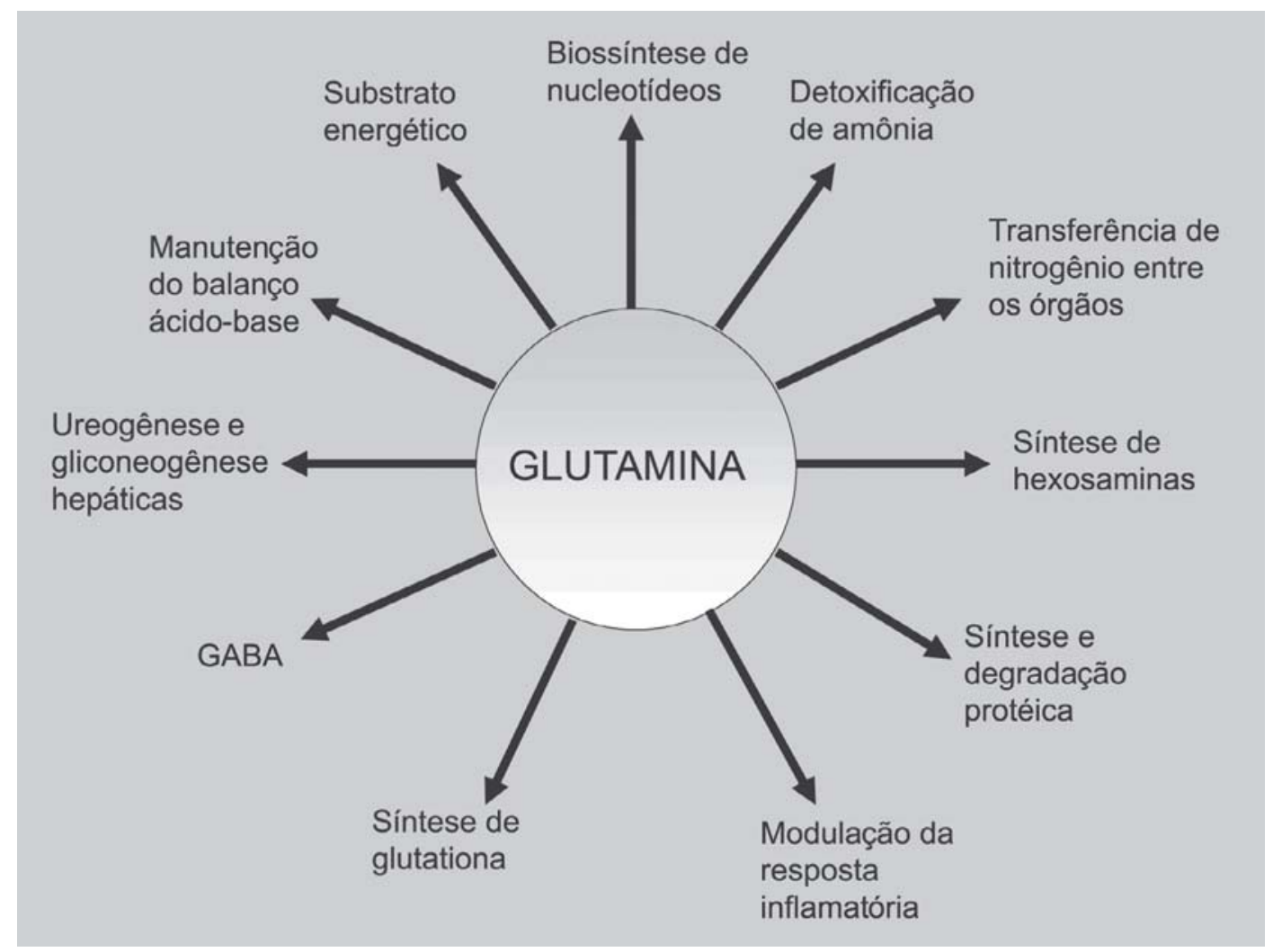

FIGURA 1 - Funções da glutamina. Modificado de Huang et al. (2003). (GABA = ácido gama-aminobutírico).

da enzima glutamina sintetase, que catalisa a conversão de glutamina a partir de amônia e glutamato, na presença de ATP. Por outro lado, tecidos que são primariamente consumidores de glutamina - células da mucosa intestinal, leucócitos e células do túbulo renal — contêm elevada atividade da enzima glutaminase, responsável pela hidrólise da glutamina e sua conversão em glutamato e amônia (Walsh et al., 1998). Sob certas condições, tal como reduzido aporte de carboidratos, o fígado pode tornar-se um sítio consumidor de glutamina (Rowbottom, Keast, Morton, 1996; Rogero, Tirapegui, 2000; Rogero et al., 2006).

As concentrações plasmática e tecidual de glutamina estão diminuídas em situações clínicas e catabólicas, tais como: traumas, queimaduras, sepse, pós-operatório, diabetes não-controlado e após exercício exaustivo. Durante estas circunstâncias, a diminuição da concentração plasmática de glutamina ocorre devido à taxa de captação e utilização deste aminoácido por diversos tecidos ser superior à taxa de síntese e liberação pelo músculo esquelético (Parry-Billings et al., 1989; Mittendorfer et al., 1999; Rogero et al., 2002). Essas situações levaram à modificação da classificação da glutamina de aminoácido não essencial ou dispensável para aminoácido condicionalmente essencial ou condicionalmente indispensável (Smith, 1990).
Neonatos são particularmente vulneráveis a infecções bacterianas, fato que é decorrente de deficiências intrínsecas da sua resposta imune, sendo a septicemia uma das principais causas de morte em crianças. O processo infeccioso pode ocasionar diminuição da concentração plasmática e tecidual de glutamina, fato que pode provocar prejuízo à funcionalidade de leucócitos em neonatos (Newsholme et al., 1999). Cabe ainda destacar que neonatos estão sujeitos a intenso crescimento, o que faz com que apresentem maior turnover protéico, fenômeno que pode acarretar em maior utilização de glutamina (Ball, Hardy, 2002). Uma vez que a glutamina é um substrato indispensável para enterócitos e células do sistema imune e que neonatos estão sujeitos a inúmeros estresses fisiológicos, sugere-se que a glutamina seja um aminoácido condicionalmente essencial nessa fase da vida (Neu, Shenoy, Chakrabarti, 1996).

\section{Fluxo transplacentário de glutamina e glutamato}

O transporte e o metabolismo da glutamina e do glutamato durante o desenvolvimento fetal possuem características únicas. De todos os aminoácidos transferidos para o feto, a glutamina é o aminoácido transferido em 
maior concentração (Neu, 2001). Estudos realizados em ovelhas durante a gestação estimam que cerca de $19 \%$ de todo o nitrogênio captado pelo feto seja derivado da glutamina (Battaglia, 2000). A glutamina liberada na circulação fetal é oriunda tanto da circulação materna, quanto da síntese placentária (Neu, 2001). No feto, o fígado capta esse aminoácido e parte da glutamina é convertida a glutamato, o qual, em seguida, é captado pela placenta. A maior parte desse glutamato é oxidada na placenta, sendo o restante convertido à glutamina, reação catalisada pela enzima glutamina sintetase, e devolvido à circulação fetal (Battaglia, 2000). Cabe destacar que, aliado ao glutamato proveniente da circulação fetal, a transaminação placentária de aminoácidos de cadeia ramificada também é responsável pelo suprimento de glutamato para a placenta (Chung et al., 1998) (Figura 2).

\section{Leite materno}

Bebês amamentados ingerem glutamina por meio do leite materno, o qual contém um pool de aminoácidos livres duas a cinco vezes maior quando comparado às formulas infantis comercializadas atualmente (Agostoni et al., 2000a). Todavia, com a progressão da lactação, observa- se diminuição significativa da concentração da maioria dos aminoácidos livres presentes no leite materno, ao mesmo tempo em que a concentração de glutamina e glutamato que representa $50 \%$ do total de aminoácidos livres no leite materno - aumenta aproximadamente 20 e 2,5 vezes, respectivamente, durante os três primeiros meses de lactação (Agostoni et al., 2000a; Agostoni et al., 2000b). Além disso, estudos demonstram que tanto no colostro quanto no leite materno, dos aminoácidos ligados à proteína, glutamina e glutamato representam aqueles de maior concentração (Davis, Fiorotto, Reeds, 1993; Davis et al., 1994; Wu, Knabe, 1994). Cabe ressaltar que a concentração de glutamina livre do leite materno é, aproximadamente, 10 vezes superior àquela encontrada em fórmulas infantis (Agostoni et al., 2000a).

Ao nascer, o fluxo transplacentário de glutamina é interrompido e o bebê passa a depender de sua síntese endógena e do fornecimento exógeno de glutamina. No entanto, neonatos pré-termo (PT) e com baixo peso ao nascer (BPN), freqüentemente, recebem apenas nutrição parenteral total nas primeiras semanas de vida, a qual não contém glutamina. Cabe ainda destacar que esses bebês possuem pouca massa muscular e, portanto, uma vez que o músculo esquelético é o principal tecido de síntese,

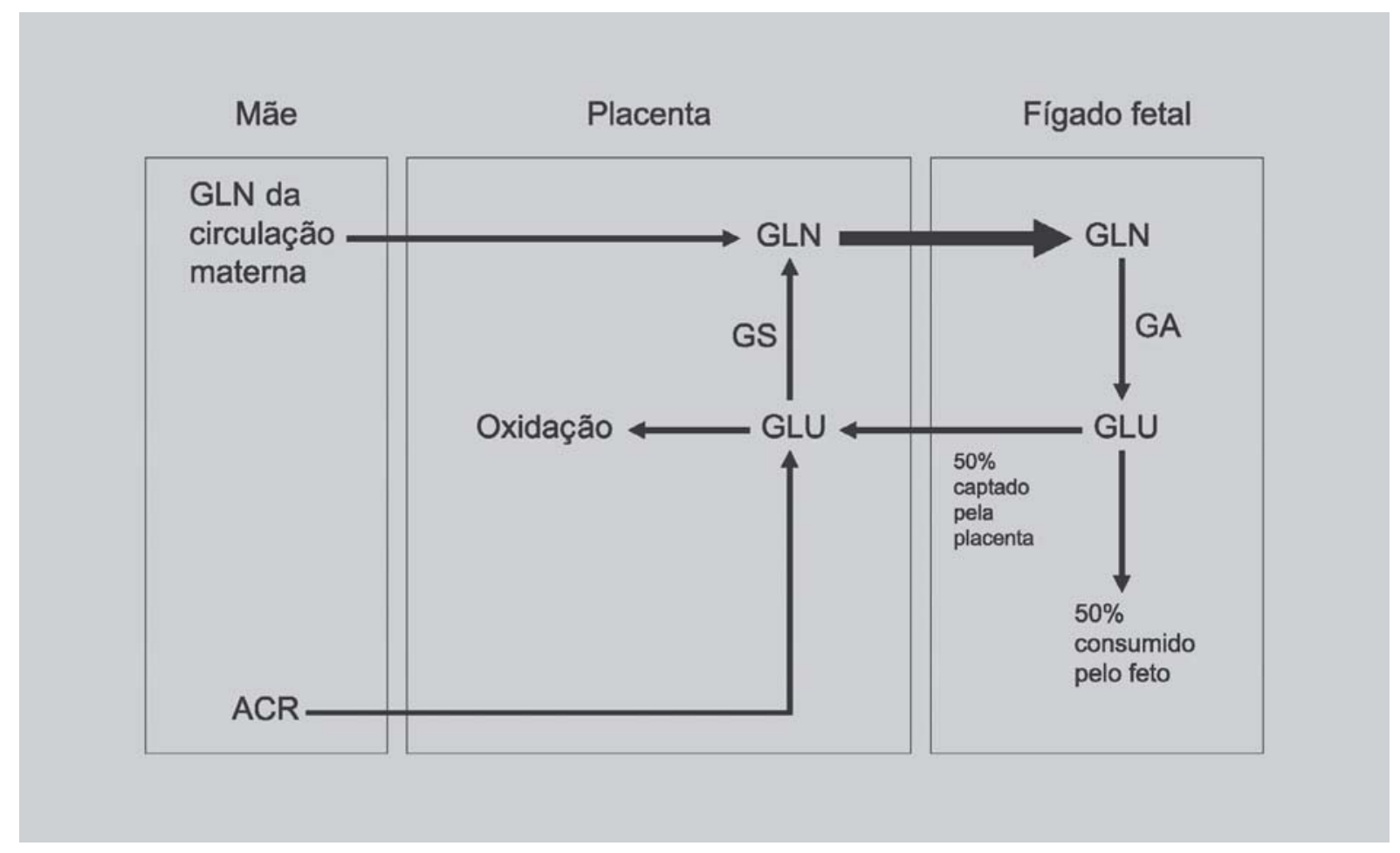

FIGURA 2 - Transferência transplacentária de glutamina e glutamato: fluxo de glutamina e glutamato entre mãe, placenta e feto. Modificado de Neu (2001). (ACR = aminoácidos de cadeia ramificada; GLU = glutamato; GLN = glutamina; GS $=$ glutamina sintetase $; \mathrm{GA}=$ glutaminase $)$. 
armazenamento e liberação de glutamina, seus estoques de glutamina são limitados. Dessa forma, é possível inferir que neonatos $\mathrm{PT}$ e com BPN recebendo nutrição parenteral total estão sob grande risco de sofrer depleção de seus estoques de glutamina, o que pode acarretar em prejuízo da função imune e da integridade intestinal.

\section{Suplementação parenteral com glutamina}

Apesar de estar envolvida em inúmeros processos metabólicos e ser o aminoácido transferido em maior concentração para o feto, a glutamina não é adicionada rotineiramente à mistura de aminoácidos de fórmulas parenterais comerciais. Este fato deve-se à limitada solubilidade da glutamina em água $\left(35 \mathrm{~g} / \mathrm{L}\right.$ de água, a $\left.20^{\circ} \mathrm{C}\right)$ (Furst, 2001), bem como à possível neurotoxicidade decorrente dos produtos da decomposição desse aminoácido: ácido piroglutâmico e amônia (Furst, Albers, Stehle, 1990). Uma alternativa que possibilita a adição de glutamina a fórmulas parenterais consiste na utilização de dipeptídeos contendo glutamina, como o L-alanil-L-glutamina, que permanece estável durante a esterilização com calor e é altamente solúvel em água (568 $\mathrm{g} / \mathrm{L}$ ) (Furst, 2001). Todavia, há escassez de estudos envolvendo a suplementação com dipeptídeos contendo glutamina para neonatos PT e com BPN.

Estudos demonstram que a administração de glutamina por via parenteral atua, principalmente, no metabolismo protéico do músculo esquelético (Parimi, Kalhan, 2007). A glutamina estimula a síntese e inibe a degradação protéica, o que poderia contribuir para a obtenção de um balanço protéico muscular menos negativo em situações catabólicas (Hammarqvist et al., 1989; Millward, Jepson, Omer, 1989). Em neonatos PT e com BPN, o efeito da suplementação parenteral com glutamina no metabolismo protéico foi avaliado por dois estudos (Des Robert et al., 2002; Kalhan et al., 2005), nos quais os bebês foram aleatoriamente selecionados para receber suplementação com glutamina intravenosa ou uma mistura de aminoácidos isonitrogenada isenta de glutamina. No protocolo utilizado por Des Robert et al. (2002), utilizou-se a suplementação aguda (24 horas) com $0,5 \mathrm{~g}$ de glutamina $/ \mathrm{kg} / \mathrm{dia}$ em neonatos PT e com BPN com quatro dias de vida. O grupo suplementado apresentou maior concentração plasmática de glutamina e menor degradação protéica e oxidação de leucina, entretanto, não foi observada melhora no balanço protéico em resposta à suplementação (Tabela I).

No estudo de Kalhan et al. (2005), uma dose maior de glutamina foi utilizada $(0,6 \mathrm{~g} / \mathrm{kg} / \mathrm{dia})$, bem como tempo de suplementação mais prolongado (72 a 120 horas) (Tabela I). Corroborando os resultados de Des Robert et al. (2002), no estudo de Kalhan et al. (2005) foi constatada diminuição da degradação protéica, concomitantemente à redução da síntese de novo de glutamina, em resposta à suplementação, $\mathrm{o}$ que sugere efeito inibitório da glutamina exógena sobre sua síntese. Por outro lado, não foi observada diferença na concentração plasmática de glutamina entre os grupos.

Em relação aos ensaios clínicos, os efeitos da suplementação parenteral com glutamina para neonatos PT e com BPN permanecem controversos. Nesse contexto, Lacey et al. (1996) observaram que a administração intravenosa de glutamina resultou em diminuição do tempo de ventilação mecânica e da nutrição parenteral total, bem como do tempo necessário para atingir a nutrição enteral exclusiva em neonatos com peso ao nascer menor que $800 \mathrm{~g}$. No entanto, esses resultados não foram confirmados em um estudo multicêntrico, no qual 1.433 neonatos

TABELA I - Suplementação parenteral com glutamina e metabolismo protéico

\begin{tabular}{lcccc}
\hline & \multicolumn{2}{c}{ Des Robert et al. $(2002)$} & \multicolumn{2}{c}{ Kalhan et al. (2005) } \\
\hline & Controle & $\begin{array}{c}\text { Suplementado } \\
\text { com GLN }\end{array}$ & Controle & $\begin{array}{c}\text { Suplementado } \\
\text { com GLN }\end{array}$ \\
\hline $\mathrm{n}$ & 7 & 6 & 10 & 10 \\
Peso ao nascer $(\mathrm{g})$ & $1.130 \pm 300$ & $1.085 \pm 200$ & $1.119 \pm 282$ & $1.003 \pm 275$ \\
Suplementação $(\mathrm{h})$ & - & 24 & - & $72-120$ \\
Concentração plasmática de GLN $(\mu \mathrm{mol} / \mathrm{L})$ & $503 \pm 83$ & $629 \pm 94 *$ & $450 \pm 140$ & $470 \pm 122$ \\
Ingestão de GLN $(\mu \mathrm{mol} / \mathrm{kg} / \mathrm{h})$ & - & 141 & - & 171 \\
Ingestão de LEU $(\mu \mathrm{mol} / \mathrm{kg} / \mathrm{h})$ & $79 \pm 10$ & $42 \pm 9 *$ & 134 & 107 \\
Ta GLN $(\mathrm{E})(\mu \mathrm{mol} / \mathrm{kg} / \mathrm{h})$ & - & - & $521 \pm 98$ & $372 \pm 66^{*}$ \\
Ta do carbono LEU $(\mu \mathrm{mol} / \mathrm{kg} / \mathrm{h})$ & $315 \pm 40$ & $240 \pm 45 *$ & $371 \pm 73$ & $324 \pm 60$ \\
Ta fenilalanina $(\mathrm{E})(\mu \mathrm{mol} / \mathrm{kg} / \mathrm{h})$ & - & - & $98 \pm 24$ & $62 \pm 17 *$ \\
\hline
\end{tabular}

Adaptado de Parimi, Kalhan (2007). Dados apresentados como média \pm desvio padrão. * ${ }^{*}<0,05$. ( $\mathrm{E}=$ endógeno; GLN $=$ glutamina $; \mathrm{LEU}=$ leucina; $\mathrm{Ta}=$ taxa de aparecimento $)$. 
com BPN foram aleatoriamente distribuídos para receber nutrição parenteral suplementada com uma solução de aminoácidos contendo $20 \%$ de glutamina $(1,4 \mathrm{~g} / 100 \mathrm{~mL}$ de solução) ou uma mistura isonitrogenada de aminoácidos. A suplementação resultou em aumento de $30 \%$ da concentração plasmática de glutamina ao final do protocolo no grupo suplementado. Apesar do aumento da concentração plasmática de glutamina sugerir aumento da disponibilidade desse aminoácido para os tecidos, não foi observada redução na incidência de sepse tardia, mortalidade, enterocolite necrosante (EN) e do tempo de hospitalização ou ventilação mecânica (Poindexter et al., 2004). Cabe ressaltar que, para que os grupos recebessem misturas de aminoácidos isonitrogenadas, o grupo suplementado com glutamina recebeu menor quantidade dos outros aminoácidos, incluindo os essenciais, fato este que pode ter interferido nos resultados observados.

Cabe enfatizar que nenhum dos estudos utilizando suplementação parenteral com glutamina observou toxicidade decorrente da suplementação (Des Robert et al., 2002; Kalhan et al., 2005; Lacey et al., 1996; Poindexter et al., 2004).

\section{Suplementação enteral com glutamina}

A nutrição enteral para neonatos PT e com BPN representa um desafio, já que a demanda metabólica é alta, mas a administração da dieta enteral é limitada devido à imaturidade do trato digestório, o que aumenta o risco de intolerância alimentar e do desenvolvimento de EN (Neu, Weiss, 1999). Sendo assim, por vezes, opta-se pela nutrição parenteral total, a qual é isenta de glutamina. Nesse contexto, Becker et al. (2000) observaram que neonatos que apresentaram EN tinham concentração plasmática de glutamina extremamente baixa 10 dias antes do aparecimento dos sinais clínicos dessa doença, indicando que a baixa concentração de glutamina pode predispor o surgimento de EN. De fato, alguns estudos clínicos demonstram melhora da tolerância à dieta enteral em neonatos PT e com BPN suplementados, via enteral, com glutamina; entretanto, não foi observada redução da incidência de EN em resposta à suplementação (Neu et al., 1997; Vaughn et al., 2003).

A dieta enteral fornece glutamina por meio das proteínas, entretanto, a quantidade fornecida é pequena, visto que a dieta enteral é limitada nas primeiras semanas de vida (Kuhn, Stehle, Furst, 1996). Dessa forma, neonatos PT ou com BPN submetidos à nutrição enteral recebem pequena quantidade de glutamina durante esse período, fato que estimulou a realização de ensaios clínicos acerca da suplementação enteral com glutamina nessa população.
Estudos em seres humanos adultos mostram que a glutamina administrada via enteral é consumida, principalmente, pela mucosa intestinal e, portanto, apenas uma parte atinge a circulação sangüínea (Windmueller, Spaeth, 1975). Em bebês PT e com BPN com cinco e seis semanas de vida, a nutrição enteral enriquecida com glutamina $(0,6$ $\mathrm{g} / \mathrm{kg} / \mathrm{dia}$ ) não resultou em alterações na taxa de aparecimento de glutamina no sangue, ao passo que se observou aumento da síntese de uréia. Esses resultados sugerem que a glutamina administrada, via enteral, foi completamente metabolizada pelo intestino e, portanto, não atingiu a circulação sangüínea (Parimi et al., 2004). Não obstante, em outro estudo (Darmaun et al., 1997), apenas cerca de $46 \%$ da glutamina administrada junto à nutrição enteral $(0,2 \mathrm{~g} / \mathrm{kg} / \mathrm{dia})$ foram extraídas pelo intestino durante o processo de absorção em neonatos PT e com BPN com 10 dias de vida.

Em contrapartida, cabe ressaltar que além de ter acesso à glutamina presente no lúmen intestinal, que atravessa a membrana apical do enterócito, o intestino também pode captar a glutamina presente no sangue arterial por meio da membrana basolateral do enterócito (Windmueller, Spaeth, 1975). Dessa forma, é possível inferir que a glutamina administrada via enteral é amplamente metabolizada pela mucosa intestinal e, portanto, o principal efeito da suplementação enteral com glutamina ocorrerá no intestino, tanto em relação à sua integridade e permeabilidade, quanto ao seu componente imune (Parimi, Kalhan, 2007).

\section{Mecanismos de ação da glutamina no intestino}

Além de ser responsável pela digestão e absorção de nutrientes, o trato digestório representa o maior tecido imune do organismo (Huang, Shao, Neu, 2003). Aliado a isso, o trato digestório é o principal tecido de captação e metabolismo da glutamina, que é consumida primariamente pelas células da mucosa intestinal, que apresentam elevado turnover no organismo de indivíduos normais (Lacey, Wilmore, 1990; Rogero, Tirapegui, 2003).

A glutamina é indispensável ao intestino, visto que é o principal substrato energético para as células epiteliais do intestino delgado e é precursora de purinas e pirimidinas, que são utilizadas em altas taxas por células de rápido turnover, como enterócitos. Adicionalmente, a glutamina doa nitrogênio para a síntese de hexosaminas - componentes de glicoproteínas e aminoaçúcares —, que são importantes para a manutenção da integridade intestinal (Neu, Shenoy, Chakrabarti, 1996; Neu, 2001).

Alguns estudos mostram que a glutamina modula a resposta inflamatória no intestino (Li et al., 2004b; Liboni et al., 2005). Esse fato é de extrema importância, uma vez 
que, em bebês PT, a resposta inflamatória exacerbada do epitélio intestinal, após colonização bacteriana, pode levar ao aumento da expressão de mediadores pró-inflamatórios como a interleucina (IL)-8, que estimula a migração de leucócitos polimorfonucleares, na fase inicial da inflamação, o que, ao longo do tempo, pode levar à lesão tecidual local e também de outros órgãos (De Dooy, Mahieu, Van Bever, 2001). Há evidências de que a resposta inflamatória exacerbada está envolvida na patogênese de diversas doenças como a doença pulmonar crônica (De Dooy, Mahieu, Van Bever, 2001), a leucomalácia periventricular (Rezaie, Dean, 2002) e a EN (Precioso, Proença, 2002), que representam complicações presentes em neonatos PT.

Há alguns mecanismos propostos para explicar de que forma a glutamina regularia a resposta inflamatória originada no intestino e a subseqüente propagação desta para outros órgãos. Dentre os mecanismos propostos estão: aumento da capacidade antioxidante do intestino por meio do aumento da síntese de glutationa, diminuição da apoptose de células intestinais, preservação da integridade da barreira intestinal e modulação direta da resposta inflamatória (Neu, Li, 2007).

Nesse contexto, verificou-se que a suplementação enteral com glutamina resultou em aumento da concentração de glutamina livre e da relação glutationa reduzida (GSH)/ glutationa oxidada (GSSG) no duodeno de cães após três dias de jejum (Humbert et al., 2007). Uma vez que não foi observado aumento da síntese de GSH, sugere-se que a glutamina promoveu a preservação dos estoques de glutationa por poupar a utilização e/ou degradação da mesma, o que pode ser devido ao aumento da síntese de NADPH secundária ao aumento da disponibilidade de $\alpha$ cetoglutarato, derivado do metabolismo do glutamato, fato que poderia favorecer a redução da GSH. Em outro estudo (Domeneghini et al., 2004), a suplementação com glutamina diminuiu a susceptibilidade à apoptose de enterócitos e aumentou a altura dos vilos em suínos após o desmame. Além disso, a suplementação com glutamina e nucleotídeos atuou sinergicamente, estimulando a proliferação celular intestinal.

Outro mecanismo proposto para a modulação da inflamação pela glutamina refere-se à manutenção da integridade das junções intercelulares, visto que alterações na barreira paracelular podem permitir a passagem de antígenos que estimulariam a resposta inflamatória $(\mathrm{Neu}$, Li, 2007). Um estudo in vitro com células CACO-2 (células intestinais), em meio de cultura isento de glutamina, demonstrou diminuição da expressão de proteínas das tight junctions (Li et al., 2004a).

Li et al. (2004b) observaram que a glutamina diminui a resposta inflamatória induzida por lipopolissacarídeo
(LPS) - componente da membrana externa de bactérias Gram-negativas - no intestino. O estímulo induzido pelo LPS atua por intermédio de receptores do tipo Toll (TLR), presentes na membrana plasmática de células do sistema imune como macrófagos, o que promove uma cascata de eventos que culminam na translocação do fator de transcrição denominado fator nuclear- $\mathrm{\kappa B}(\mathrm{NF}-\kappa \mathrm{B})$ para o interior do núcleo celular e conseqüente ativação da transcrição de diversos genes dependentes do $\mathrm{\kappa B}$, como genes de citocinas pró-inflamatórias. No citoplasma de células não estimuladas, o NF- $\kappa B$ encontra-se na forma inativa devido a sua associação com proteínas denominadas inibidores do $\kappa \mathrm{B}$ (IкB). Com a finalidade de elucidar o mecanismo pelo qual a glutamina poderia diminuir a expressão de citocinas próinflamatórias, Liboni et al. (2005) estimularam células intestinais fetais, in vitro, com LPS. As células em meio de cultura isento de glutamina apresentaram menor síntese de IKB, o que, possivelmente, possibilitou maior translocação

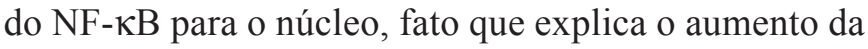
expressão de mediadores pró-inflamatórios (IL-8) nessas células (Liboni et al., 2005).

\section{Estudos clínicos com suplementação enteral com glutamina}

Em razão da glutamina desempenhar papel importante na manutenção da integridade intestinal e na função imune, alguns estudos clínicos foram realizados para avaliar se a suplementação enteral com glutamina em neonatos PT e com BPN diminuiria a incidência de morbidades infecciosas como a sepse nosocomial, ou seja, a sepse adquirida em meio hospitalar, condição que ocorre freqüentemente em neonatos PT e com BPN durante a fase de convalescença de sua hospitalização, e que está associada com significativa taxa de morbidade e mortalidade (Makhoul et al., 2002).

Neu et al. (1997) observaram menor incidência de sepse nosocomial em neonatos PT e com BPN recebendo dieta enteral suplementada com $0,3 \mathrm{~g}$ de glutamina $/ \mathrm{kg} / \mathrm{dia}$, durante 28 dias, em relação ao grupo controle. Adicionalmente, a porcentagem de linfócitos expressando na sua membrana plasmática a molécula HLA-DR, a qual está envolvida na ativação de linfócitos, não se alterou no grupo suplementado com glutamina, ao passo que, no grupo controle, houve aumento no percentual dessas células na terceira e quarta semanas de vida em relação ao início do protocolo. Além disso, a contagem absoluta e o percentual de células natural killer CD16 e CD56 apresentou menor aumento no grupo suplementado em comparação ao grupo controle. Esses resultados indicam que o grupo suplementado com glutamina sofreu menor estimulação da resposta imune, que pode ser secundária à redução da translocação de antígenos, o que, por sua 
vez, acarreta em menor incidência de sepse. Todavia, é relevante destacar que o número de participantes do estudo foi pequeno $(n=68)$, e que o grupo controle não recebeu uma dieta isonitrogenada e, portanto, os resultados encontrados podem ter sido influenciados pela maior ingestão de proteína (até 0,31 $\mathrm{g} / \mathrm{kg} / \mathrm{dia}$ ) e de nitrogênio pelo grupo suplementado. Em outro estudo, o mesmo protocolo de suplementação foi utilizado $(0,3$ g de glutamina $/ \mathrm{kg} /$ dia por 28 dias), entretanto, o grupo controle recebeu uma mistura isonitrogenada contendo alanina. Assim como no estudo de Neu et al. (1997), a incidência de sepse no grupo de PT e com BPN que recebeu nutrição enteral suplementada com glutamina foi menor em relação ao controle (van den Berg et al., 2005) (Tabela II).

Neonatos prematuros podem ter permeabilidade intestinal aumentada devido à imaturidade de seu trato digestório, o que, por sua vez, aumenta a susceptibilidade à translocação bacteriana, o que pode predispor à ocorrência de sepse. Dessa forma, van den Berg et al. (2006) investigaram se a suplementação enteral com glutamina resultaria em melhora da integridade intestinal, o que levaria à diminuição da permeabilidade intestinal, fato que poderia justificar a menor incidência de sepse observada no grupo suplementado com este aminoácido. Contudo, não foi observada redução da permeabilidade intestinal em resposta à suplementação enteral com $0,3 \mathrm{~g}$ de glutamina $/ \mathrm{kg} / \mathrm{dia}$. Apesar de algumas limitações metodológicas, os autores concluíram que os resultados positivos encontrados nos estudos anteriores acerca da suplementação enteral com glutamina envolvem outros aspectos, como modulação direta da resposta inflamatória intestinal pela glutamina (van den Berg et al., 2006).

A relação entre a incidência de sepse e a suplementação enteral com glutamina também foi investigada por um ensaio clínico, multicêntrico, com 649 participantes (Vaugh et al., 2003). Ao contrário dos estudos anteriores, não foi observada diferença na incidência de sepse em neonatos suplemen- tados com glutamina $(0,3 \mathrm{~g} / \mathrm{kg} / \mathrm{dia})$, por 28 dias, em comparação ao grupo placebo (água estéril). No entanto, a dose suplementada foi baseada no peso ao nascimento e não foi ajustada para o peso real. Conseqüentemente, a quantidade de glutamina fornecida pode ter sido insuficiente, devido ao rápido ganho de peso pós-natal. Além disso, similarmente aos estudos realizados por Van den Berg et al. (2005) e Van den Berg et al. (2006), parte dos bebês em ambos os grupos recebeu glutamina por meio do leite materno e, portanto, o grupo controle não foi privado de suprimento exógeno de glutamina. A concentração de glutamina no leite materno não foi dosada em nenhum dos estudos e, portanto, não é possível estimar a quantidade de glutamina ingerida pelo grupo controle.

Em relação à capacidade da glutamina de modular a resposta inflamatória, Vaughn et al. (2003) observaram que neonatos com BPN suplementados via enteral com glutamina apresentaram menor incidência de hemorragia intraventricular e leucomalácia periventricular, distúrbios associados com estados pró-inflamatórios. Todavia, não foi encontrada redução da incidência de outras doenças relacionadas à inflamação, como doença pulmonar crônica e EN.

Outros parâmetros avaliados pelos ensaios clínicos envolvendo suplementação enteral com glutamina são tolerância à dieta enteral, crescimento físico, EN e tempo de hospitalização. A suplementação enteral com glutamina promoveu melhora da tolerância à dieta enteral em um dos estudos (Neu et al., 1997). No entanto, não tem sido observada melhora no crescimento físico, na diminuição da incidência de EN ou no tempo de hospitalização em bebês suplementados com glutamina (Neu et al., 1997; Vaughn et al., 2003; Van den Berg et al., 2005). Da mesma forma que os estudos envolvendo suplementação parenteral com glutamina, nenhum dos estudos realizados com suplementação enteral com glutamina observou toxicidade decorrente da suplementação (Neu et al., 1997; Vaughn et al., 2003; Van den Berg et al., 2005; Van den Berg et al., 2006).

TABELA II - Suplementação enteral com glutamina

\begin{tabular}{lcccccc}
\hline & \multicolumn{2}{c}{ Neu et al. (1997) } & \multicolumn{2}{c}{ Vaughn et al. (2003) } & \multicolumn{2}{c}{ Van den Berg et al. (2005) } \\
\hline & $\begin{array}{c}\text { Controle } \\
(\mathrm{n}=33)\end{array}$ & $\begin{array}{c}\text { Suplementado } \\
(\mathrm{n}=35)\end{array}$ & $\begin{array}{c}\text { Controle } \\
(\mathrm{n}=335)\end{array}$ & $\begin{array}{c}\text { Suplementado } \\
(\mathrm{n}=314)\end{array}$ & $\begin{array}{c}\text { Controle } \\
(\mathrm{n}=50)\end{array}$ & $\begin{array}{c}\text { Suplementado } \\
(\mathrm{n}=52)\end{array}$ \\
\hline Peso ao nascer (g) & $965 \pm 29$ & $939 \pm 34$ & $900 \pm 190$ & $890 \pm 200$ & $1160 \pm 300$ & $1180 \pm 400$ \\
Leite materno (\%) & - & - & 52 & 48 & $60 * *$ & $60 * *$ \\
GLN (g/kg/dia) & - & 0,3 & - & 0,3 & - & 0,3 \\
Dieta enteral exclusiva (dias) & 13 & 13 & $3,5 \pm 2$ & $3,5 \pm 2$ & $9(4-23)$ & $10(4-17)$ \\
Tolerância à dieta enteral & & Melhora & & Sem efeito & & Sem efeito \\
Sepse, n (\%) & $10(33)$ & $4(11)^{*}$ & $113(34)$ & $97(31)$ & $38(76)$ & $26(50)^{*}$ \\
\hline A & &
\end{tabular}

Adaptado de Parimi, Kalhan, 2007. Dados apresentados como média \pm desvio padrão. * $\mathrm{p}<0,01$; ** Bebês sob aleitamento materno exclusivo. (GLN = glutamina). 


\section{CONCLUSÃO}

Assim como em diversos estados clínicos, é possível que a glutamina seja um aminoácido condicionalmente essencial para neonatos PT e com BPN e, portanto, a suplementação com glutamina poderia promover benefícios para este grupo. Além disso, os estudos clínicos realizados em neonatos PT e com BPN, envolvendo suplementação enteral ou parenteral com glutamina, não observaram toxicidade em resposta à suplementação. De fato, alguns ensaios clínicos demonstraram benefícios da suplementação com este aminoácido em neonatos PT e com BPN. Em contrapartida, esses resultados não foram confirmados em estudos multicêntricos e, portanto, até o presente momento, não há evidências suficientes que justifiquem a utilização rotineira da suplementação com glutamina para neonatos PT e com BPN. É possível que diferenças na quantidade de aminoácidos oferecida para os grupos estudados e a heterogeneidade da população em questão sejam parcialmente responsáveis pelas discrepâncias encontradas. Adicionalmente, cabe destacar que há necessidade de mais estudos com a finalidade de esclarecer os mecanismos de ação da glutamina no intestino, bem como qual via de administração (parenteral ou enteral) é mais vantajosa para neonatos PT e com BPN.

\section{ABSTRACT \\ Enteral and parenteral supplementation with glutamine in preterm and low-birth-weight neonates}

Glutamine is the most abundant amino acid found in the blood and skeletal muscle, and is the principal energetic substrate for cells with a high turnover, such as enterocytes and leucocytes. Furthermore, glutamine is the most important amino acid that is passed to the foetus via the placenta, and together with glutamate, is the most abundant amino acid in maternal milk. Preterm infants suffer an abrupt interruption in the supply of glutamine via the placenta, which leads to an exclusive dependence on the endogenous synthesis or the exogenous supply of this amino acid. Preterm neonates (PT) as well as lowbirth-weight neonates ( $L B W$ ) frequently receive only total parenteral nutrition during their first weeks of life, which contains no glutamine. It must be pointed out that these infants present low muscular mass and that therefore, their stock of glutamine is limited. Because PT and BPN neonates are subject to intensive growth periods and numerous physiological stresses, it is possible that glutamine is a conditionally essential nutrient in this stage of life, thus giving foundation for the pursuit of studies aiming at the evaluation of the possible clinical benefits of enteral and parenteral supplementation with glutamine in PT and BPN neonates.

UNITERMS: Glutamine. Supplementation. Neonate. Enteral nutrition. Parenteral nutrition.

\section{AGRADECIMENTOS}

Os autores agradecem ao Conselho Nacional de Desenvolvimento Científico e Tecnológico (CNPq), pelas bolsas outorgadas, e à Fundação de Amparo à Pesquisa do Estado de São Paulo (FAPESP), pelo auxílio financeiro.

\section{REFERÊNCIAS BIBLIOGRÁFICAS}

AGOSTONI, C.; CARRATU, B.; BONIGLIA, C.; RIVA, E.; SANZINI, E. Free amino acid content in standard infant formulas: comparison with human milk. J. Am. Coll. Nutr., v.19, p.434-438, 2000a.

AGOSTONI, C.; CARRATU, B.; BONIGLIA, C.; RIVA, E.; SANZINI, E. Free glutamine and glutamic acid increase in milk through a three-month lactation period. J. Pediatr. Gastroenterol. Nutr., v.31, p.508-512, 2000 b.

BALL, P. A.; HARDY, G. Glutamine in pediatrics: where next? Nutrition, v.18, p.451-454, 2002.

BATTAGLIA, F. C. Glutamine and glutamate exchange between the fetal liver and the placenta. J. Nutr., v.130, p.974S-977S, 2000.

BECKER, R. M.; WU, G.; GALANKO, J. A.; CHEN, W.; MAYNOR, A. R.;BOSE, C.L.; RHOADS, J.M. Reduced serum amino acid concentrations in infants with necrotizing enterocolitis. J. Pediatr., v.137, p.785-793, 2000.

CHUNG, M.; TENG, C.; TIMMERMAN, M.; MESCHIA, G.; BATTAGLIA, F. C. Production and utilization of amino acids by ovine placenta in vivo. Am. J. Physiol., v.274, p.E13-E22, 1998.

DAVIS, T. A.; FIOROTTO, M. L.; REEDS, P. J. Amino acid compositions of body and milk protein change during the suckling period in rats. J. Nutr., v.123, p.947-956, 1993.

DAVIS, T. A.; NGUYEN, H. V.; GARCIA-BRAVO, R.; FIOROTTO, M. L.; JACKSON, E. M.; REEDS, P. J. Amino acid composition of the milk of some mammalian species changes with stage of lactation. Br. J. Nutr., v.72, p.845-853, 1994. 
DARMAUN, D.; ROIG, J. C.; AUESTAD, N.; SAGER, B. K.; NEU, J. Glutamine metabolism in very low birth weight infants. Pediatr. Res., v.41, p.391-396, 1997.

DE DOOY, J.J.; MAHIEU, L.M.; VAN BEVER, H.P. The role of inflammation in the development of chronic lung disease in neonates. Eur. J. Pediatr., v.160, p.457-463, 2001.

DES ROBERT, C.; LE BACQUER, O.; PILOQUET, H.; ROZE, J. C.; DARMAUN, D. Acute effects of intravenous glutamine supplementation on protein metabolism in very low birth weight infants: a stable isotope study. Pediatr. Res., v.51, p.87-93, 2002.

DOMENEGHINI, C.; DI GIANCAMILLO, A.; SAVOINI, G.; PARATTE, R.; BONTEMPO, V.; DELL'ORTO, V. Structural patterns of swine ileal mucosa following Lglutamine and nucleotide administration during the weaning period. An histochemical and histometrical study. Histol. Histopathol., v.19, p.49-58, 2004.

FURST, P. New developments in glutamine delivery.J. Nutr., v.131, p.2562S-2568S, 2001.

FURST, P.; ALBERS, S.; STEHLE, P. Glutamine-containing dipeptides in parenteral nutrition. JPEN, v.14, p.118S124S, 1990.

HAMMARQVIST, F.; WERNERMAN, J.; ALI, R.; VON DER DECKEN, A.; VINNARS, E. Addition of glutamine to total parenteral nutrition after elective abdominal surgery spares free glutamine in muscle, counteracts the fall in muscle protein synthesis, and improves nitrogen balance. Ann. Surg., v.209, p.455-461, 1989.

HUANG, Y.; SHAO, X. M.; NEU, J. Immunonutrients and neonates. Eur. J. Pediatr., v.162, p.122-128, 2003.

HUMBERT, B.; NGUYEN, P.; MARTIN, L.; DUMON, H.; VALLETTE, G.; MAUGĖRE, P.; DARMAUN, D. Effect of glutamine on glutathione kinetics in vivo in dogs. J. Nutr. Biochem., v.18, p.10-16, 2007.

KALHAN, S. C.; PARIMI, P. S.; GRUCA, L. L.; HANSON, R. W. Glutamine supplement with parenteral nutrition decreases whole body proteolysis in low birth weight infants. J. Pediatr., v.146, p.642-647, 2005.
KUHN, K. S.; STEHLE, P.; FURST, P. Glutamine content of protein and peptide-based enteral products. JPEN, v.20, p.292-295, 1996.

LACEY, J. M.; CROUCH, J. B.; BENFELL, K.; RINGER, S. A.; WILMORE, C. K.; MAGUIRE, D.; WILMORE, D. W. The effects of glutamine-supplemented parenteral nutrition in premature infants. JPEN, v.20, p.74-80, 1996.

LACEY, J. M.; WILMORE, D. W. Is glutamine a conditionally essential amino acid? Nutr. Rev., v.48, p.297309, 1990.

LI, N.; LEWIS, P.; SAMUELSON, D.; LIBONI, K.; NEU, J. Glutamine regulates caco-2 cell tight junction proteins. Am. J. Physiol. Gastrointest. Liver Physiol., v.287, p.G726-G733, 2004a.

LI, N.; LIBONI, K. C.; FANG, M. Z.; SAMUELSON, D.; LEWIS, P.; PATEL, R.; NEU, J. Glutamine decreases lipopolysaccharide-induced intestinal inflammation in infant rats. Am. J. Physiol. Gastrointest. Liver Physiol., v.286, p.G914-G921, 2004b.

LIBONI, K. C.; LI, N.; SCUMPIA, P. O.; NEU, J. Glutamine modulates LPS-induced IL-8 production through IKB/NF$\kappa \mathrm{B}$ in human fetal and adult intestinal epithelium. J. Nutr., v. 135, p.245-51, 2005.

MAKHOUL, I. R.; SUJOV, P.; SMOLKIN, T.; LUSKY, A.; REICHMAN, B. Epidemiological, clinical, and microbiological characteristics of late-onset sepsis among very low birth weight infants in Israel: a national survey. Pediatrics, v.109, p. 34-39, 2002.

MILLWARD, D. J.; JEPSON, M. M.; OMER, A. Muscle glutamine concentration and protein turnover in vivo in malnutrition and in endotoxemia. Metabolism, v.38, p.613, 1989.

MITTENDORFER, B.; GORE, D. C.; HERNDON, D. N.; WOLFE, R. R. Accelerated glutamine synthesis in critically ill patients cannot maintain normal intramuscular free glutamine concentration. JPEN, v.23, p.243-250, 1999.

NEU, J. Glutamine in the fetus and critically ill low birth weight neonate: metabolism and mechanism of action. $J$. Nutr., v.131, p.2585S-2589S, 2001. 
NEU, J.; LI, N. Pathophysiology of glutamine and glutamate metabolism in premature infants. Curr. Opin. Clin. Nutr. Metab. Care, v.10, p.75-79, 2007.

NEU, J.; ROIG, J. C.; MEETZE, W. H.; VEERMAN, M.; CARTER, C.; MILLSAPS, M.; BOWLING, D.; DALLAS, M. J.; SLEASMAN, J.; KNIGHT, T.; AUESTAD, N. Enteral glutamine supplementation for very low birth weight infants decreases morbidity. $J$. Pediatr., v.131, p.691-699, 1997.

NEU, J.; SHENOY, V.; CHAKRABARTI, R. Glutamine nutrition and metabolism: where do we go from here? FASEB J., v.10, p.829-837, 1996.

NEU, J.; WEISS, M. D. Necrotizing enterocolitis: pathophysiology and prevention. JPEN, v.23, p.S13-17, 1999.

NEWSHOLME, P.; CURI, R.; CURI, T. C. P.; MURPHY, C. J.; GARCIA, C.; MELO, M. P. Glutamine metabolism by lymphocytes, macrophages, and neutrophils: its importance in health and disease. J. Nutr. Biochem., v.10, p.316-324, 1999.

PARIMI, P. S.; DEVAPATLA, S.; GRUCA, L. L.; AMINI, S. B.; HANSON, R. W.; KALHAN, S. C. Effect of enteral glutamine or glycine on whole-body nitrogen kinetics in very-low-birth-weight infants. Am. J. Clin. Nutr., v.79, p.402-409, 2004.

PARIMI, P. S.; KALHAN, S. C. Glutamine supplementation in the newborn infant. Semin. Fetal Neonatal Med., v.12, p.19-25, 2007.

PARRY-BILLINGS, M.; LEIGHTON, B.; DIMITRIADIS, G.; VASCONCELOS, P. R. L.; NEWSHOLME, E. A. Skeletal muscle glutamine metabolism during sepsis in the rat. Int. J. Biochem., v.21, p.419-423, 1989.

POINDEXTER, B. B.; EHRENKRANZ, R. A.; STOLL, B. J.; WRIGHT, L. L.; POOLE, W. K.; OH, W.; BAUER, C. R.; PAPILE, L. A.; TYSON, J. E.; CARLO, W. A.; LAPTOOK, A. R.; NARENDRAN, V.; STEVENSON, D. K.; FANAROFF, A. A.; KORONES, S. B.; SHANKARAN, S.; FINER, N. N.; LEMONS, J. A. Parenteral glutamine supplementation does not reduce the risk of mortality or late-onset sepsis in extremely low birth weight infants. Pediatrics, v.113, p.1209-1215, 2004.
PRECIOSO, A. R.; PROENÇA, R. S. M. Necrotizing enterocolitis, pathogenesis and the protector effect of prenatal corticosteroids. Rev. Hosp. Clín. Fac. Med. S. Paulo, v.57, p.243-248, 2002.

REZAIE, P.; DEAN A. Periventricular leukomalacia, inflammation and white matter lesions within the developing nervous system. Neuropathology; v.22, p. 106-132, 2002.

ROGERO, M. M.; TIRAPEGUI, J. Aspectos nutricionais sobre glutamina e exercício físico. Nutrire, v.25, p.101$126,2003$.

ROGERO, M. M.; PEDROSA, R. G.; TIRAPEGUI, J.; CASTRO, I.A.; PIRES, I. S. O. Effect of L-alanyl-Lglutamine supplementation on plasma, liver and muscle concentration of glutamine in rats submitted exhaustive exercise. Nutrition, v.22, p.564-571, 2006.

ROGERO, M. M.; TIRAPEGUI, J. Aspectos atuais sobre glutamina, atividade física e sistema imune. Rev. Bras. Cienc. Farm., v.36, p.201-212, 2000.

ROGERO, M. M.; TIRAPEGUI, J.; PEDROSA, R. G.; CASTRO, I. A.; PIRES, I. S. O.; OLIVEIRA, A. A. M.; SALGADO, M. M.; PINTO, A. R.; UEDA, M. Efeito da suplementação com L-alanil-L-glutamina sobre a resposta de hipersensibilidade do tipo tardio em ratos submetidos ao treinamento intenso. Rev. Bras. Cienc. Farm., v.38, p.487-497, 2002.

ROGERO, M. M.; TIRAPEGUI, J.; PEDROSA, R. G.; CASTRO, I. A.; PIRES, I. S. O. Plasma and tissue glutamine response to acute and chronic supplementation with L-glutamine and L-alanyl-L-glutamine in rats. Nutr. Res., v.24, p.261-270, 2004.

ROWBOTTOM, D. G.; KEAST, D.; MORTON, A. R. The emerging role of glutamine as an indicator of exercise stress and overtraining. Sports Med., v.21, p.80-97, 1996.

SMITH, R. J. Glutamine metabolism and its physiologic importance. JPEN, v.14, p.40S-44S, 1990.

VAN DEN BERG, A.; FETTER, W. P.; WESTERBEEK, E. A.; VAN DER VEGT, I. M.; VAN DER MOLEN, H. R.; VAN ELBURG, R. M. The effect of glutamine-enriched enteral nutrition on intestinal permeability in very-lowbirth-weight infants: a randomized controlled trial. JPEN, v.30, p.408-414, 2006. 
VAN DEN BERG, A..; VAN ELBURG, R. M.; WESTERBEEK, E. A.; TWISK, J. W.; FETTER, W. P. Glutamine-enriched enteral nutrition in very-low-birthweight infants and effects on feeding tolerance and infectious morbidity: a randomized controlled trial. Am.J. Clin. Nutr., v.81, p.1397-1404, 2005.

VAUGHN, P.; THOMAS, P.; CLARK, R.; NEU, J. Enteral glutamine supplementation and morbidity in low birth weight infants. J. Pediatr., v.142, p.662-668, 2003.

WALSH, N. P.; BLANNIN, A. K.; ROBSON, P. J.; GLEESON, M. Glutamine, exercise and immune function: links and possible mechanisms. Sports Med., v.26, p.177-191, 1998.
WINDMUELLER, H. G.; SPAETH, A. E. Intestinal metabolism of glutamine and glutamate from the lumen as compared to glutamine from blood. Arch. Biochem. Biophys., v.171, p.662-672, 1975.

WU, G.; KNABE, D. A. Free and protein-bound amino acids in sow's colostrum and milk. J. Nutr., v.124, p.415-424, 1994.

Recebido para publicação em 06 de julho de 2007. Aceito para publicação em 23 de novembro de 2007. 